\title{
Structural heterogeneity of the microtubule lattice
}

Charlotte Guyomar ${ }^{1}$, Siou Kuํㄹ ${ }^{1}$ John Heumann², Clément Bousquet ${ }^{1}$, Gabriel Guilloux ${ }^{1}$, Natacha Gaillard $^{3}$, Claire Heichette ${ }^{1}$, Laurence Duchesne ${ }^{1}$, Michel O. Steinmetz ${ }^{3,4}$, Romain Gibeaux ${ }^{1}$, Denis Chrétien ${ }^{1 *}$

\section{Affiliations:}

${ }^{1}$ Univ Rennes, CNRS, IGDR (Institut de Génétique et Développement de Rennes) - UMR 6290; Rennes, France.

${ }^{2}$ Departement of Molecular, Cellular and Developmental Biology, University of Colorado, Boulder, CO 80309 USA.

${ }^{3}$ Laboratory of Biomolecular Research, Division of Biology and Chemistry, Paul Scherrer Institute, Villigen, Switzerland.

${ }^{4}$ University of Basel, Biozentrum, 4056 Basel, Switzerland

*Corresponding author. Email: denis.chretien@univ-rennes1.fr

\begin{abstract}
:
Microtubules are polymers assembled from $\alpha \beta$-tubulin heterodimers. They typically display lateral $\alpha-\alpha$ and $\beta-\beta$ homotypic interactions, except at one region, called the seam, where heterotypic $\alpha-\beta$ and $\beta-\alpha$ interactions occur. Here, we decorated microtubules assembled in vitro or in cytoplasmic Xenopus egg extracts with kinesin-motor domains, and analyzed their lattice organization using dual axis cryo-electron tomography followed by segmented subtomogram averaging. In both conditions, microtubules incorporated variable protofilament and/or tubulin subunit helix start numbers. While microtubules assembled in vitro displayed variable numbers of seams, those assembled in extracts displayed preferentially one seam. The seam location varied within individual microtubules implying the presence of lattice holes. Thus, the formation of discontinuous microtubule lattices is an intrinsic property of tubulin assembly, a process that is controlled in cells.
\end{abstract}

\section{One-Sentence Summary:}

Microtubules assembled from purified tubulin form discontinuous lattices, an intrinsic property strictly controlled in cytoplasmic Xenopus egg extracts. 


\section{Main Text:}

Microtubules are tubes of about $25 \mathrm{~nm}$ in diameter, which can reach several tens of micrometers in length. They are critically involved in a variety of functions including cell motility, cell compartmentalization, and cell division (1). They are commonly described to be made of 13 parallel protofilaments that associate laterally with a $\sim 0.93 \mathrm{~nm}$ longitudinal stagger so that the $\alpha$ and $\beta$ subunits of the $\alpha \beta$-tubulin heterodimer follow left-handed, three-start lateral helices around their wall (13_3 microtubules; Fig. 1A, top) (2). The lateral contacts between tubulin subunits are thought to be predominantly homotypic ( $\alpha-\alpha$ and $\beta-\beta$, B-lattice), except at one particular region that is made of heterotypic lateral interactions called the seam $(\alpha-\beta$ and $\beta-\alpha$, A-lattice). Yet, a detailed analysis of microtubule lattice organization is still lacking. In particular, our knowledge of their structures in cells remains limited $(3,4)$. To address these issues, we decorated microtubules assembled in vitro from purified mammalian tubulin or in cytoplasmic Xenopus egg extracts with kinesin-motor domains that bind to $\beta$-tubulin (Fig. 1A, bottom), and analyzed their lattice binding pattern using cryo-electron tomography followed by sub-tomogram averaging. To this end, we specifically developed a novel segmented subtomogram averaging (SSTA) strategy, which allowed us to investigate the structural heterogeneity of individual microtubule lattices.

Microtubules were self-assembled in vitro from purified porcine brain tubulin for about one hour (fig. S1A) and kinesin-motor domains were added just before vitrification of the specimen grids into liquid ethane (fig. S1B, top). Cryo-electron tomograms were acquired using a dual-axis strategy (5) so that all microtubules could be analyzed independently of their orientation with respect to the tilt axes (fig. S1C, movie S1). We first processed entire microtubules present in the tomograms using a sub-tomogram averaging approach that retrieves small sub-volumes of about $40 \mathrm{~nm}^{3}$ in size at every kinesin-motor domain position (i.e., every $\sim 8 \mathrm{~nm}$; fig. S2A) (6). The resulting $3 \mathrm{D}$ volumes clearly revealed the protofilament number and the organization of the kinesin-motor domains around the microtubule lattice (Fig. 1B, movie S2), and hence the underlying organization of their constituent tubulin dimers (Fig. 1B, bottom right).

In agreement with previous studies $(3,7-9)$, we found that microtubules assembled in vitro from purified tubulin contained one or several A-lattice seams (fig. S3). However, we could frequently observe protofilaments with a much finer appearance where the kinesin-motor domain periodicity was partly or completely lost (Fig. 1C, movie S3). We hypothesized that the appearance of such aberrant protofilaments resulted from the averaging of regions containing kinesin-motor domain densities with regions falling in between. To explore this idea, we performed sub-tomogram averaging of short regions along microtubules (Fig. 1D-E, fig. S2B). Using this approach, we could confirm that these abnormal protofilaments originated from a mixture of regions with and without kinesin-motor domains, indicating an offset of at least one tubulin subunit along individual protofilaments in these regions (Fig. 1F). At the organizational level of the microtubule lattice, this result implies a change from one lattice type to another one within individual microtubules, and hence the presence of holes inside their walls.

Next, we wondered whether the formation of lattice discontinuities was an intrinsic property of tubulin polymerization and if such microtubule lattice defects are also present in a cellular context. Decorating microtubules with kinesin-motor domains in cells remains challenging, since it involves removing of the cell membrane with detergents, adding kinesin-motor domains, and obtaining sufficiently thin specimens that can be analyzed by electron microscopy $(3,4)$. To overcome these difficulties and to allow the analysis of a large data set of cytoplasmic 
microtubules, we took advantage of the open cellular system constituted by metaphase-arrested cytoplasmic Xenopus laevis egg extracts ((10); from here onwards referred to as "cytoplasmic extracts"). Cryo-fluorescence microscopy was used to optimize the density of microtubule asters onto electron-microscope grids (fig. S4A). Kinesin-motor domains were added to label-free extracts just before vitrification (fig. S1B, bottom) and specimens were imaged by dual-axis cryo-electron tomography (fig. S4B) followed by SSTA (Fig. 2A).

The vast majority of the microtubule segments were organized according to a 13 protofilament, three-start helix in a B-lattice configuration with one single seam (fig. S5). Yet, lattice-type transitions were observed in six cases over the 64 microtubules analyzed. These transitions systematically involved a lateral offset of the seam by one protofilament (Fig. 2B). In addition, variations in protofilament and helix start numbers were also observed such as 12_2, 12_3, 13_4 and 14_3 microtubule-lattice regions (Fig. 2C). Of note, the 12_2 and 13_4 microtubules showed a local dislocation in between two protofilaments (movie S6), which is likely a response to the excessive protofilament skewing present in these microtubules (11). The 12_2 microtubule contained two seams, while the 13_4 microtubules had no seams and hence were fully helical at the tubulin dimer level. These observations show that changes in protofilament and/or helix start numbers, as well as multiple seams and transitions in lattice types, occur within individual microtubules in a cytoplasmic environment.

The results obtained so far revealed an unexpected heterogeneity of the microtubule lattice, both when assembled in vitro from purified tubulin and in cytoplasmic extracts. However, we found marked differences between the two conditions. First, microtubules in both systems show a range of protofilament and/or helix start numbers (Fig. 2D, left): While microtubules assembled from purified tubulin are predominantly $14 \_3(64.9 \%)$ and $13 \_3(33.1 \%)$, the vast majority of those assembled in cytoplasmic extracts are $13 \_3(96.3 \%)$. Second, in both cases B-lattice contacts predominate (Fig. 2D, middle left). However, a marked difference concerns the number of seams (Fig. 2D, middle right): It can be as high as seven in microtubules assembled from purified tubulin (fig. S3:MT13) and is mainly one in cytoplasmic-extract microtubules. Third, the number and location of seams varies within individual microtubules assembled from purified tubulin, with an average lattice-type transition frequency of $3.7 \mu \mathrm{m}^{-1}$ (Fig. 2D, right), while it is only 0.1 $\mu \mathrm{m}^{-1}$ in microtubules assembled in cytoplasmic extracts $(\mathrm{p}<0.0001$, Mann-Whitney test). Finally, protofilament number and/or helix start number transitions were observed in both cases, but with no marked difference between microtubules assembled in vitro $\left(0.10 \mu \mathrm{m}^{-1}\right)$ and those assembled in cytoplasmic extracts $\left(0.07 \mu \mathrm{m}^{-1} ; \mathrm{p}=0.38\right.$, Mann-Whitney test $)$.

Changes in protofilament number, helix start number, and lattice types within individual microtubules imply the presence of holes within their lattices. Here, we have illustrated gaps of only one tubulin subunit, which is the minimum required to produce the lattice-type transitions observed in microtubules assembled from purified tubulin (Fig. 1F) and in cytoplasmic extracts (Fig. 2B). However, lattice gaps of a larger number of subunits are likely present, but seem to remain limited in size since we could not identify these holes in raw tomograms. The presence of holes within the microtubule lattice agrees with recent observations suggesting that tubulin dimer exchange is not restricted to microtubule ends, but also occurs within their wall (12). In a recent study, the lattice exchange rate of tubulin was compared to the protofilament-number transition frequency in microtubules assembled in vitro (13). It was shown that both frequencies increase with the microtubule growth rate. While it remains to be determined whether such a correlation can be made with lattice-type transitions, our results suggest that the main origin of tubulin lattice exchange sites are holes induced by such transitions. 
Changes in lattice types along individual microtubules could result from an imperfect annealing of shorter microtubules, a process known to occur in vitro (14). Yet, the high lattice-type transition frequency observed with purified tubulin would necessitate annealing of very short segments, a few tens to hundreds of $\mathrm{nm}$ in length. The average lattice-type transition frequency observed in cytoplasmic extracts could be compatible with annealing of microtubules a few $\mu \mathrm{m}$ in length. However, the fact that these transitions involved systematically a lateral seam offset of only one protofilament suggests a firm regulatory mechanism. Hence, a more plausible explanation is that these lattice discontinuities are formed during microtubule assembly (Fig. 3, movie S7). At present, all models of microtubule elongation hypothesize that tubulin engages either uniquely longitudinal interactions (Fig. 3A, step 1), or both longitudinal and lateral interactions with the growing tip of microtubules (Fig. 3A, step 2) (15). A purely longitudinal elongation process (16) can hardly explain how microtubules can vary in terms of protofilament and/or helix start numbers as well as in lattice types, and thus how holes can arise during assembly. Conversely, to account for the presence of holes of one or a few subunits in size, it is sufficient to consider that tubulin can also engage lateral interactions without longitudinal ones (Fig. 3A, step 3). Gaps of an odd number of tubulin subunits will induce lattice-type transitions (Fig. 3A, step 4-5), while those of an even number will induce no changes (Fig. 3B) and thus will not be directly detected by our analysis unless they are of sufficient size to diminish the overall density of the kinesin-motor domains in sub-tomogram averages. Hence, since both types of events are likely to occur, we may underestimate the presence of holes by at least a factor of two. In addition, a finer sampling of the microtubule lattice with shorter segments could also reveal a much higher hole frequency. Formation of lateral contacts without longitudinal ones at the seam region can also explain how the seam can vary in position by one protofilament (Fig. 3C), since this only requires that a tubulin dimer engages homotypic lateral interactions at the seam region (Fig. 3C, step 2). This event will also leave a gap of an odd number of subunits within the microtubule lattice (Fig. 3C, steps 3-4).

Our current view of microtubules organized according to a perfect pseudo-helical B-lattice interrupted by a single A-lattice seam must be revised. This is definitely the case for microtubules assembled from purified tubulin and has profound consequences for the interpretation of biochemical, biophysical, and structural results. For instance, 3D reconstruction studies will have to take into account the heterogeneity of the microtubule lattice to reach higher resolution (7). The lattice organization of cytoplasmic-extract microtubules is more in agreement with the B-lattice, single seam model. However, exceptions are also observed such as changes in protofilament and/or helix start numbers, as well as in the location of seams within individual microtubules. Therefore, our results suggest that the formation of heterogeneous microtubule lattices is an intrinsic property of tubulin polymerization, which is firmly regulated in cells. One key regulatory factor could be the $\gamma$-tubulin ring complex ( $\gamma$ TuRC), which imposes the 13 protofilament organization to a nascent microtubule (17). But how this structure is preserved during microtubule elongation remains unclear, especially if one considers a two-dimensional assembly process where the lattice can vary in terms of protofilament number, helix start number, or lattice type during elongation. Proteins of the end-binding (EB) family are other good candidates that could play a key role in regulating microtubule structure during assembly in cells. They interact with the tip of growing microtubules and bind in between protofilaments that are organized according to a B-lattice (18); they thus may favor the formation of homotypical lateral interactions during assembly. In addition, EBs have been shown to induce the formation of 13 protofilament, three-start helix microtubules (19), which could also be forced to adopt a preferential B-lattice organization. Conversely, microtubule polymerases like XMAP215, which 
act at growing microtubule ends (20) may favor lattice heterogeneities. It remains to be determined whether the concerted action of different microtubule growing-end binding proteins regulate microtubule structure and dynamics in cells (21).

1. A. Desai, T. J. Mitchison, Microtubule polymerization dynamics. Annu Rev Cell Dev Biol. 13, 83-117 (1997).

2. E. M. Mandelkow, R. Schultheiss, R. Rapp, M. Müller, E. Mandelkow, On the surface lattice of microtubules: helix starts, protofilament number, seam, and handedness. $J$ Cell Biol. 102, 1067-1073 (1986).

3. M. Kikkawa, T. Ishikawa, T. Nakata, T. Wakabayashi, N. Hirokawa, Direct visualization of the microtubule lattice seam both in vitro and in vivo. J Cell Biol. 127, 1965-1971 (1994).

4. J. R. McIntosh, M. K. Morphew, P. M. Grissom, S. P. Gilbert, A. Hoenger, Lattice structure of cytoplasmic microtubules in a cultured Mammalian cell. J Mol Biol. 394, 177-182 (2009). tomography of microtubules assembled in vitro: limits and perspectives. J Struct Biol. 181, 169178 (2013).

6. D. Zabeo, J. M. Heumann, C. L. Schwartz, A. Suzuki-Shinjo, G. Morgan, P. O. Widlund,

J. L. Höög, A lumenal interrupted helix in human sperm tail microtubules. Sci Rep. 8, 2727 (2018).

7. G. E. Debs, M. Cha, X. Liu, A. R. Huehn, C. V. Sindelar, Dynamic and asymmetric fluctuations in the microtubule wall captured by high-resolution cryoelectron microscopy. Proc Natl Acad Sci U S A. 117, 16976-16984 (2020).

8. H. Sosa, A. Hoenger, R. A. Milligan, Three different approaches for calculating the threedimensional structure of microtubules decorated with kinesin motor domains. J Struct Biol. 118, 149-158 (1997).

9. A. des Georges, M. Katsuki, D. R. Drummond, M. Osei, R. A. Cross, L. A. Amos, Mal3, the Schizosaccharomyces pombe homolog of EB1, changes the microtubule lattice. Nat Struct Mol Biol. 15, 1102-1108 (2008).

10. R. Gibeaux, R. Heald, The Use of Cell-Free Xenopus Extracts to Investigate Cytoplasmic Events. Cold Spring Harb Protoc. 2019 (2019), doi:10.1101/pdb.top097048.

11. D. Chrétien, S. D. Fuller, Microtubules switch occasionally into unfavorable configurations during elongation. J Mol Biol. 298, 663-676 (2000).

12. M. Théry, L. Blanchoin, Microtubule self-repair. Current Opinion in Cell Biology. 68, 144-154 (2021).

13. L. Schaedel, S. Triclin, D. Chrétien, A. Abrieu, C. Aumeier, J. Gaillard, L. Blanchoin, M. Théry, K. John, Lattice defects induce microtubule self-renewal. Nat Phys. 15, 830-838 (2019). 14. S. W. Rothwell, W. A. Grasser, D. B. Murphy, End-to-end annealing of microtubules in vitro. J Cell Biol. 102, 619-627 (1986).

$40 \quad$ 15. V. VanBuren, D. J. Odde, L. Cassimeris, Estimates of lateral and longitudinal bond energies within the microtubule lattice. Proc Natl Acad Sci U S A. 99, 6035-6040 (2002).

16. J. R. McIntosh, E. O’Toole, G. Morgan, J. Austin, E. Ulyanov, F. Ataullakhanov, N. Gudimchuk, Microtubules grow by the addition of bent guanosine triphosphate tubulin to the tips of curved protofilaments. Journal of Cell Biology. 217, 2691-2708 (2018). tubulin ring complex: Deciphering the molecular organization and assembly mechanism of a major vertebrate microtubule nucleator. BioEssays. n/a, 2100114. 
18. A. Akhmanova, M. O. Steinmetz, Tracking the ends: a dynamic protein network controls the fate of microtubule tips. Nat Rev Mol Cell Biol. 9, 309-322 (2008).

19. B. Vitre, F. M. Coquelle, C. Heichette, C. Garnier, D. Chrétien, I. Arnal, EB1 regulates microtubule dynamics and tubulin sheet closure in vitro. Nat Cell Biol. 10, 415-421 (2008). 20. G. J. Brouhard, J. H. Stear, T. L. Noetzel, J. Al-Bassam, K. Kinoshita, S. C. Harrison, J. Howard, A. A. Hyman, XMAP215 is a processive microtubule polymerase. Cell. 132, 79-88 (2008).

21. A. Akhmanova, M. O. Steinmetz, Control of microtubule organization and dynamics: two ends in the limelight. Nat Rev Mol Cell Biol. 16, 711-726 (2015). 
bioRxiv preprint doi: https://doi.org/10.1101/2021.07.14.452321; this version posted July 14,2021. The copyright holder for this preprint (which

was not certified by peer review) is the author/funder, who has granted bioRxiv a license to display the preprint in perpetuity. It is made available under aCC-BY-NC-ND 4.0 International license.

A
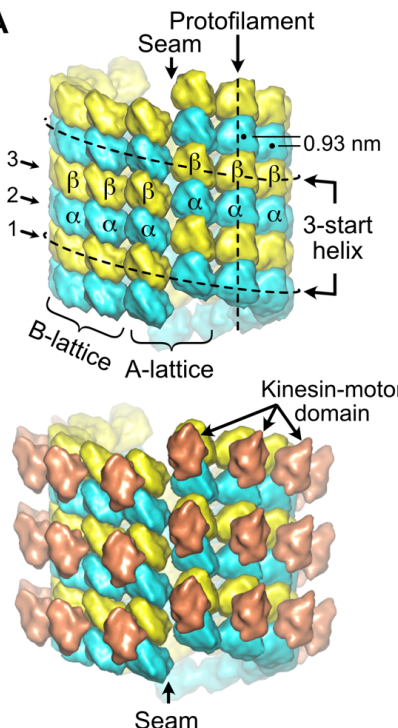

C

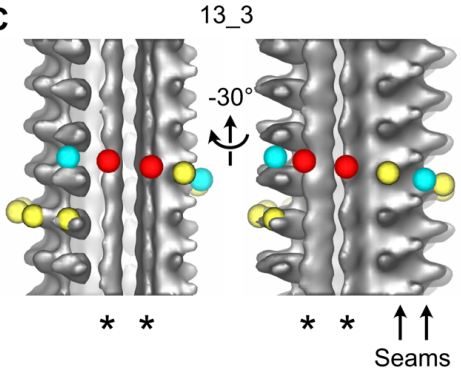

$\mathbf{E}$

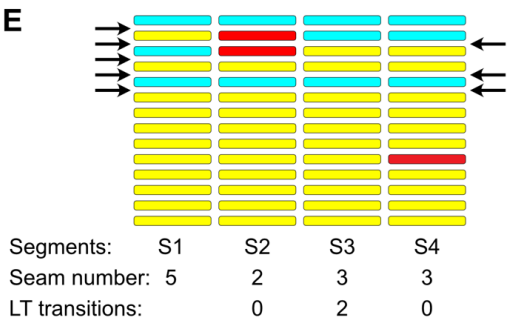

$\mathbf{F} \quad \mathrm{s} 1$

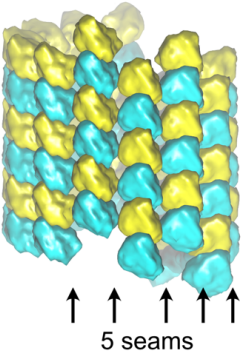

S2

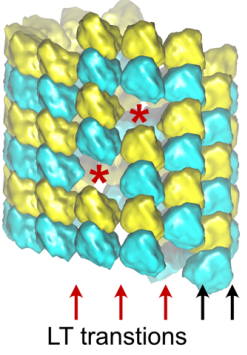

B
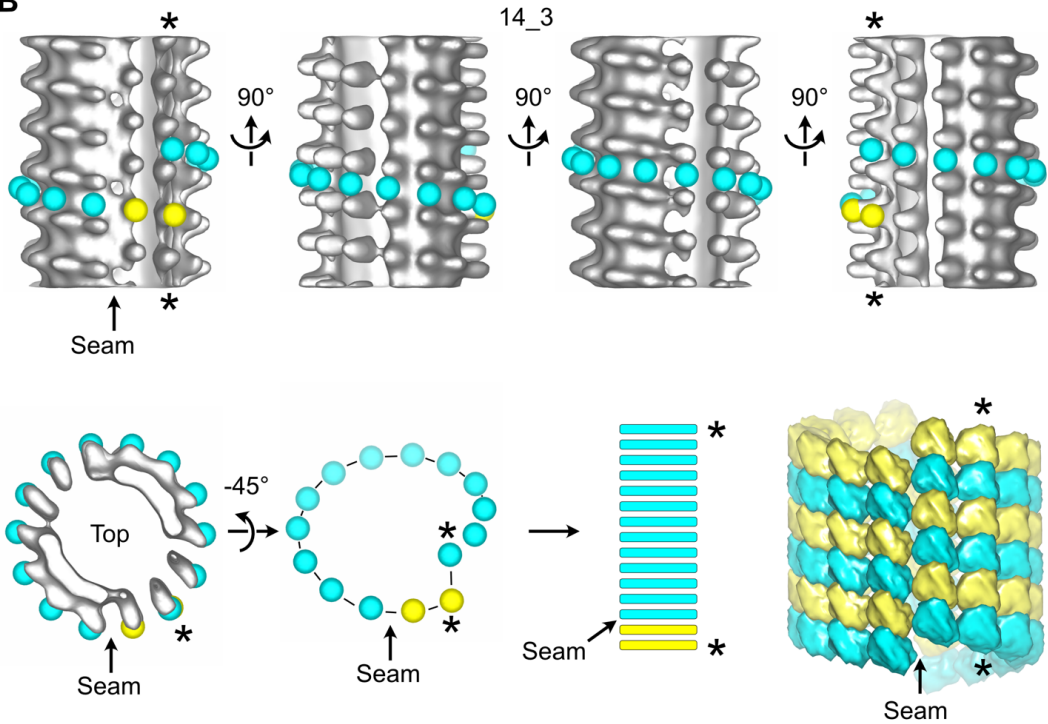

D
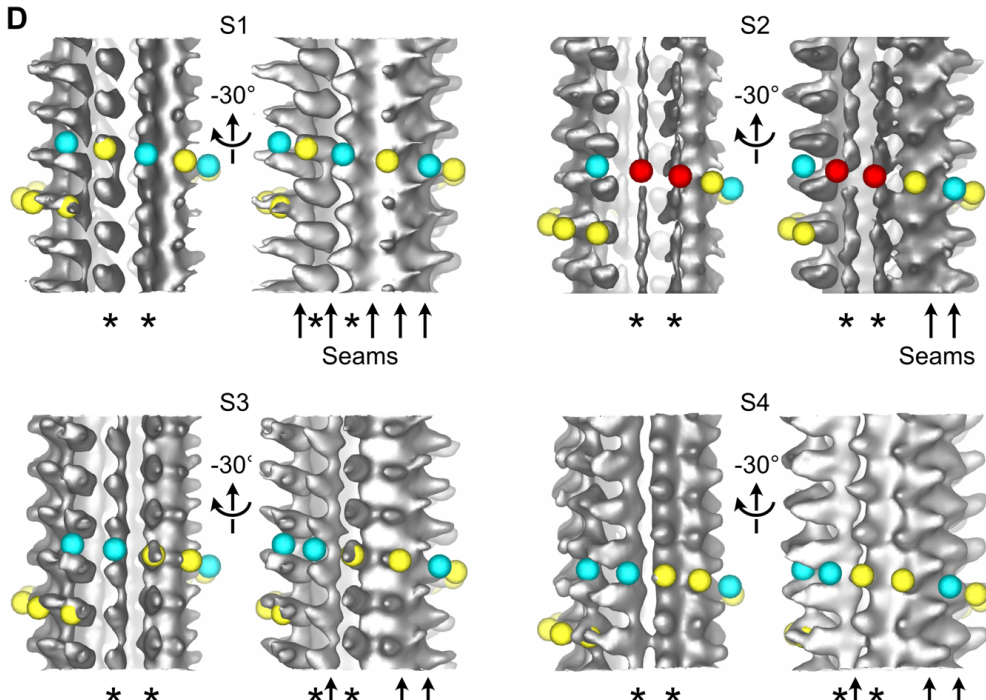

3
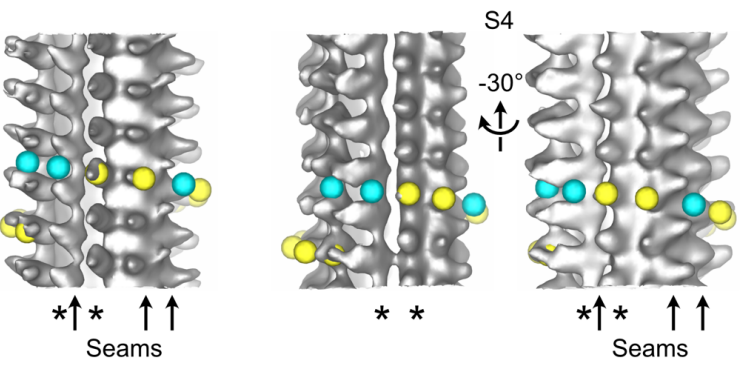

S3

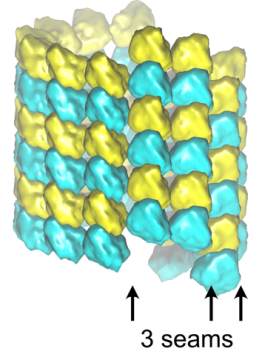


Fig. 1 Microtubules assembled from purified tubulin contain several seams whose number and location vary within their lattices.

(A) Organization of tubulin within microtubules. Top: $\alpha \beta$-tubulin heterodimers ( $\alpha$ in cyan, $\beta$ in yellow) alternate head-to-tail along protofilaments, 13 of which associate laterally to form the microtubule wall. The lateral association of the $\alpha$ - and $\beta$-tubulin subunits describe a left-handed three-start helix around the microtubule wall. They form preferentially homotypic B-lattice lateral interactions, except at one region, called the seam, where A-lattice interactions occur. Bottom: Decoration of microtubules with kinesin-motor domains (orange) highlights the tubulin dimer lattice. (B) Average of a $1390.4 \mathrm{~nm}$ long 14_3 microtubule assembled in vitro from purified tubulin and reconstructed in 3D by sub-tomogram averaging (fig. S3, MT3, movie S2). Top: Views turned $90^{\circ}$ around the Y-axis. Yellow spheres have been placed onto the kinesinmotor domain densities and cyan spheres in between. They follow the left-handed, three-start helix of the microtubule lattice. A kinesin-motor domain is juxtaposed laterally to a lack of density along the three-start helix at the seam, producing a characteristic zig-zag pattern. Bottom: Top view of the volume (left), sphere model turned $45^{\circ}$ around the $\mathrm{X}$-axis (middle left), flat representation of the model (middle right), and 3D model of the underlying tubulin dimer lattice (right). The stars $\left(^{*}\right)$ highlight the same protofilament in each representation. (C) Average of a $1327.2 \mathrm{~nm}$ long 13_3 microtubule (fig. S3, MT5, movie S3) displaying two aberrant protofilaments $(*)$, and two adjacent seams (arrows in the $-30^{\circ}$ view). Red spheres have been placed on top of the aberrant protofilaments. (D) Segmented sub-tomogram averaging of the microtubule in (C). The microtubule has been divided into 4 segments $331.8 \mathrm{~nm}$ in length, and sub-tomogram averages have been performed on each segment (S1 to S4). The two aberrant protofilaments in (C) are well resolved in S1, S3 and S4, while they still display an aberrant shape in S2. The lattice organization of these protofilaments must be offset by at least one tubulin subunit between S1 and S3. Hence, S2 constitutes a transition zone where kinesin-motor domain densities and absence of densities have been averaged. (E) Flat representation of the lattice organization within segments S1 to S4. S1 contains 5 seams while S3 and S4 contain 3 seams (arrows). Two lattice type transitions occur between S1 and S3, and S4 contains an aberrant protofilament (movie S3). A finer segmentation of the microtubule at $165.9 \mathrm{~nm}$ revealed an additional lattice-type transition in this region (fig. S3, MT5, between segments S5 and S7). (F) Lattice organization of S1, S2 and S3. Holes (red stars) of a minimum size of one tubulin subunit (or an odd number) must be present in segment S2 to account for the offset in lattice-type organization between S1 and S3. Dark arrows point to seams, and red arrows in S2 to lattice-type transitions. 
bioRxiv preprint doi: https://doi.org/10.1101/2021.07.14.452321; this version posted July 14, 2021. The copyright holder for this preprint (which was not certified by peer review) is the author/funder, who has granted bioRxiv a license to display the preprint in perpetuity. It is made available under aCC-BY-NC-ND 4.0 International license.

A
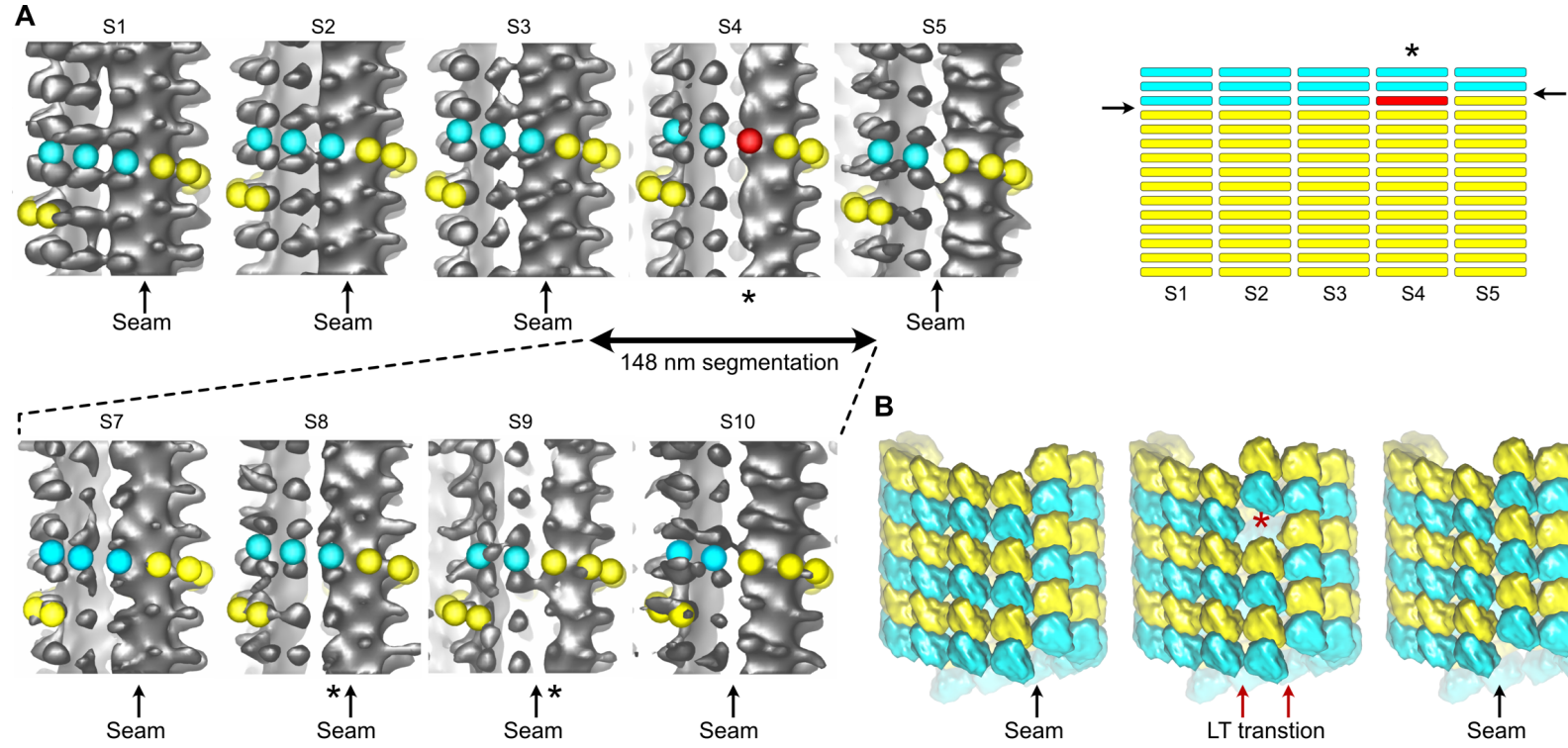

S10

\section{' $\mathrm{B}$}

C

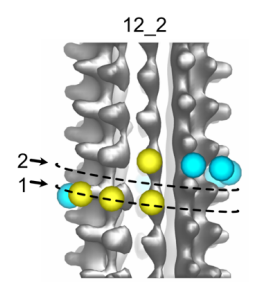

$-\left(\rightarrow 90^{\circ}\right.$
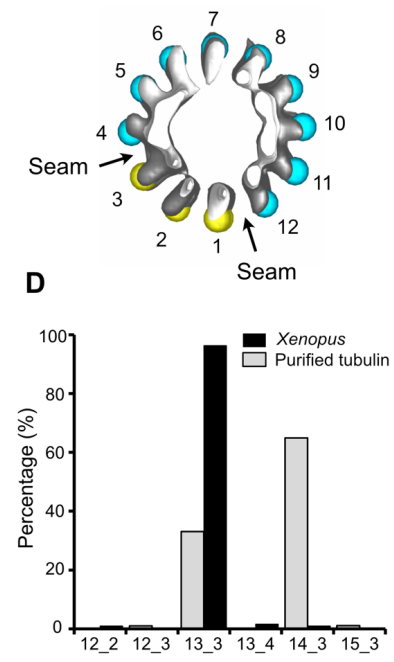

$\uparrow^{*}$
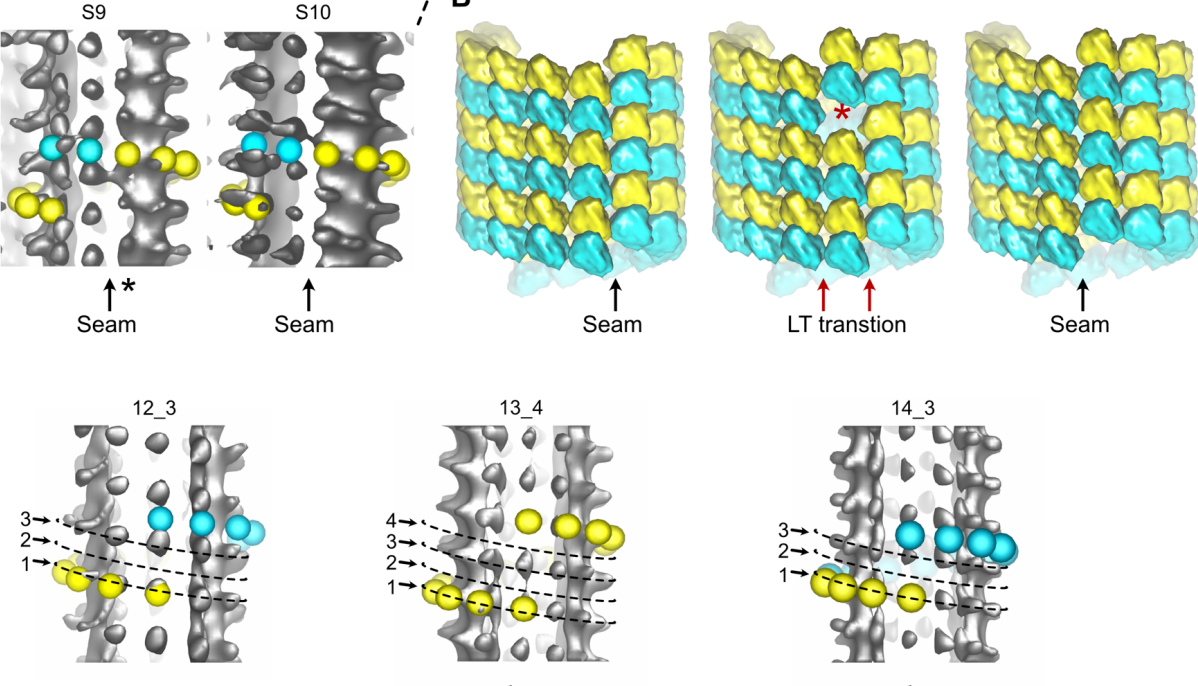

$-\leftrightarrow 90^{\circ}$
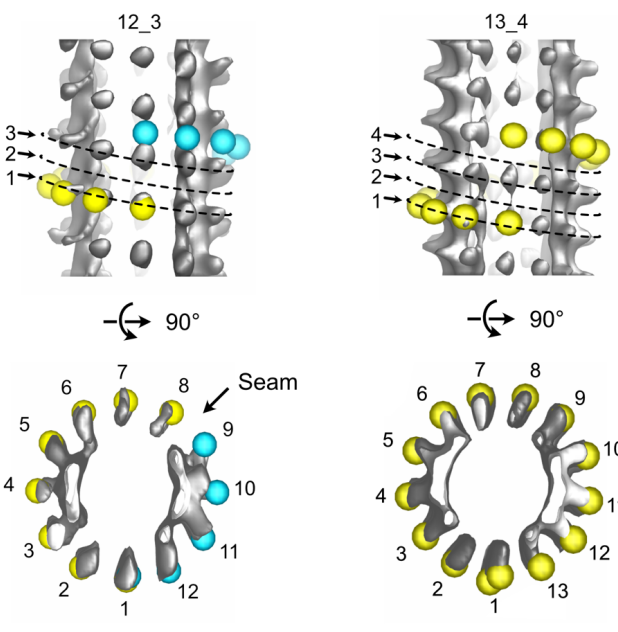

$-\leftrightarrow 90^{\circ}$
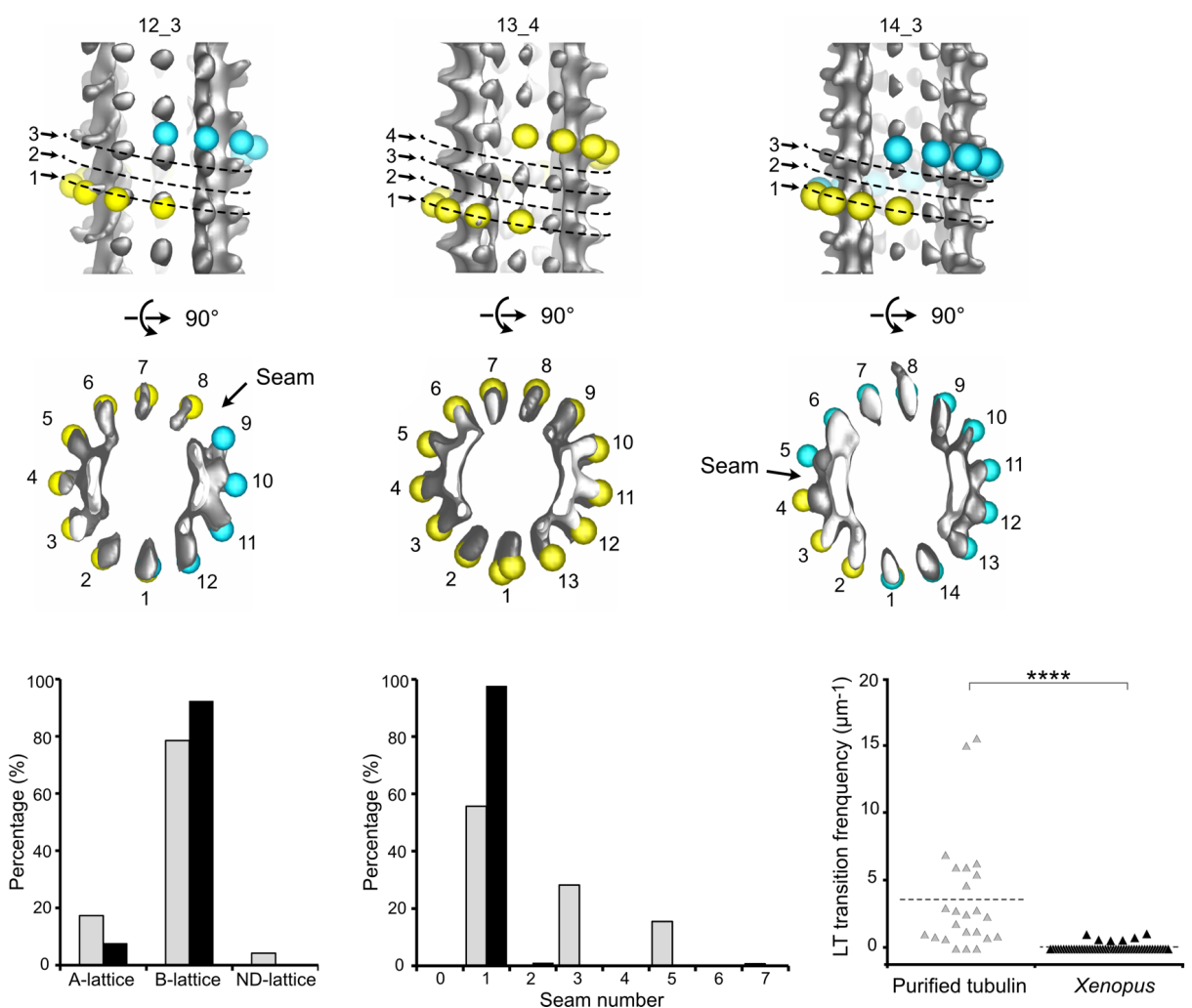
Fig. 2 Microtubules assembled in Xenopus egg cytoplasmic extracts contain preferentially a unique seam whose location varies within their lattices.

(A) Top left: Sub-tomogram averages of five $310.8 \mathrm{~nm}$ long segments of a 13 _ 3 microtubule (original length $1628 \mathrm{~nm}$, movie S5). S4 contains an aberrant protofilament (*), and the seam (arrow) moves laterally by one protofilament from S3 to S5. Top right: flat representation of the five segments. Bottom: Sub-tomogram averages of $148 \mathrm{~nm}$ long segments. The microtubule has been segmented into 11 segments (fig. S5, MT2). Only S7 to S10 are shown, corresponding to a region that encompass S3 to S5 in the $310.8 \mathrm{~nm}$ segmentation. The lattice-type transition occurs from S8 to S9, and no aberrant protofilament is seen with this finer segmentation. (B) 3D models of the tubulin lattice before (left), during (middle) and after (right) the transition. The lateral offset in seam position requires a longitudinal offset of a minimum of one tubulin subunit to account for the lattice-type transition observed in (A). (C) Microtubules with different protofilament and/or helix start numbers (movie S6). Left: 12_2 with two seams (fig. S5, MT9). Middle left: 12_3 with a unique seam (fig. S5, MT32). Middle right: 13 _ 4 with no seam (fig. S5, MT7). Right: $14 \_3$ with one seam (fig. S5, MT32). (D) Comparison between microtubules assembled from purified porcine brain tubulin or in cytoplasmic extracts (grey bars: porcine brain microtubules, dark bars: cytoplasmic-extract microtubules). Left: Microtubule types. Middle left: Percentages of A- and B-lattices. ND: not determined. Middle right: Percentage of seam numbers. Right: Lattice-type transition frequency. Short microtubules with less than four segments were excluded from the analysis. Comparison was performed using the Mann-Whitney test $(\mathrm{p}<0.0001)$. 
bioRxiv preprint doi: https://doi.org/10.1101/2021.07.14.452321; this version posted July 14, 2021. The copyright holder for this preprint (which

was not certified by peer review) is the author/funder, who has granted bioRxiv a license to display the preprint in perpetuity. It is made available under aCC-BY-NC-ND 4.0 International license.

A

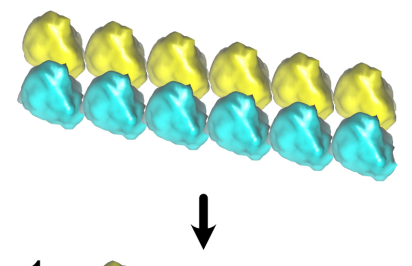

1

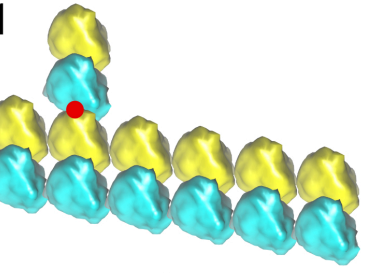

$\downarrow$

2

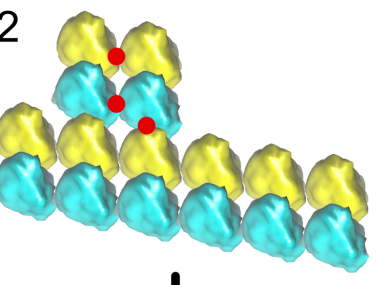

3
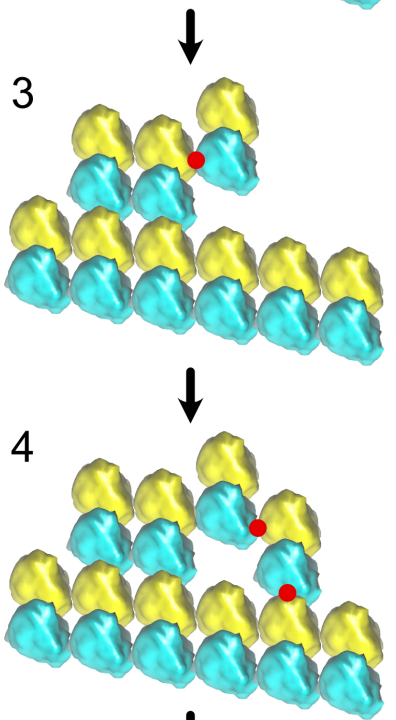

:

5

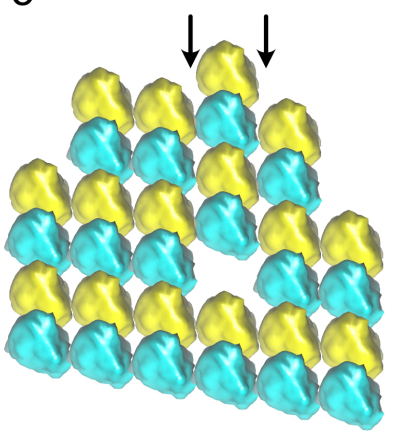

B

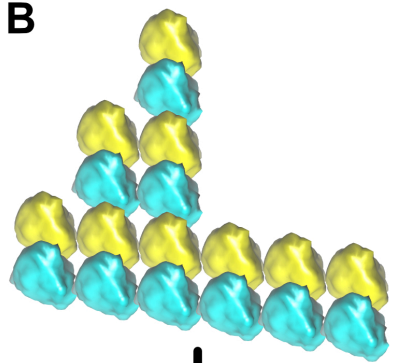

1

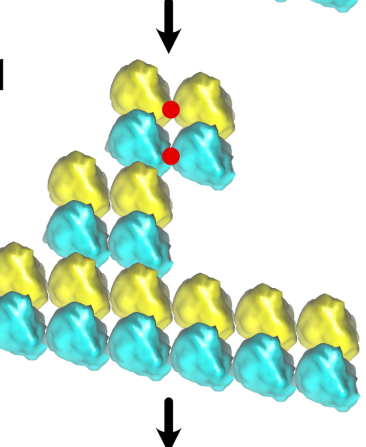

2

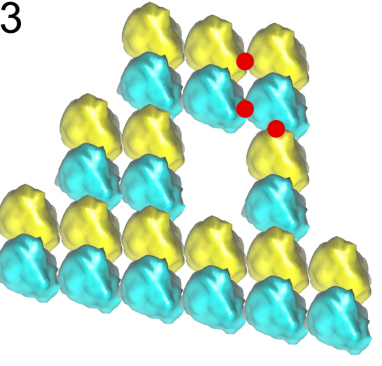

!

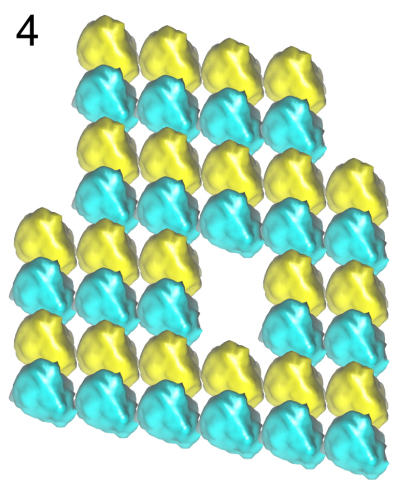

C
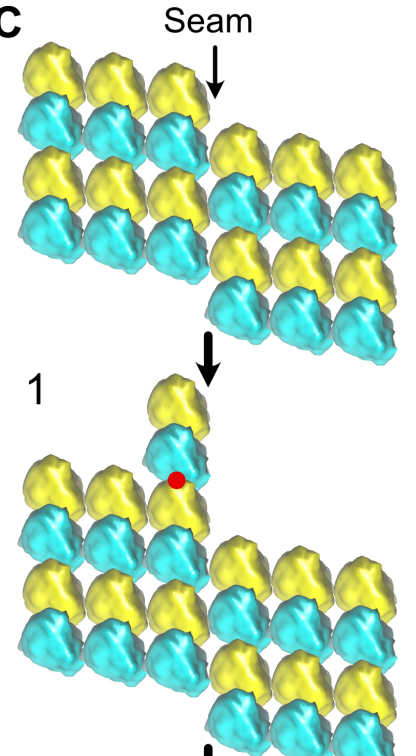

2
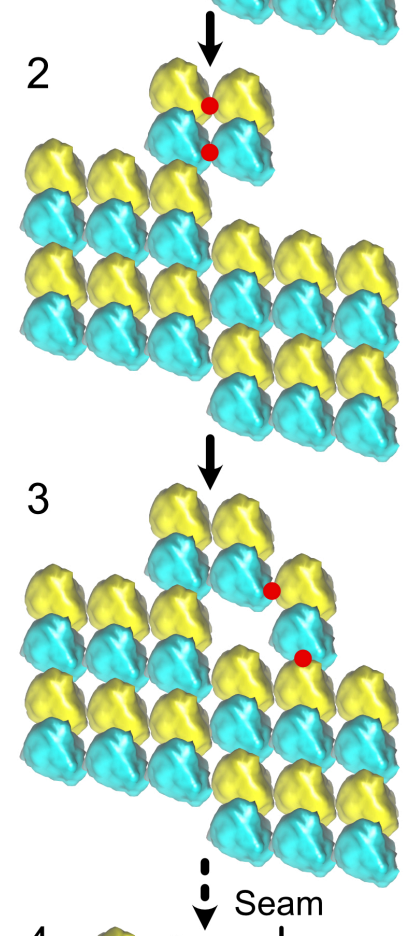

4

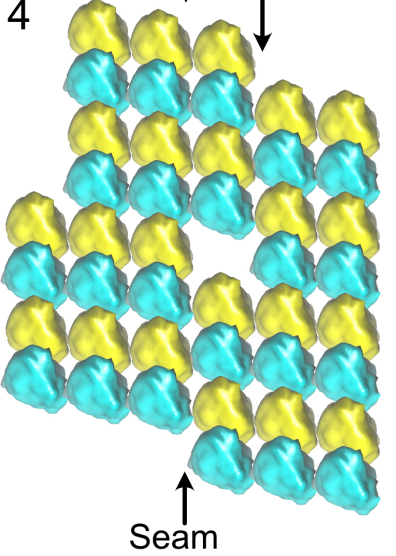


Fig. 3 Molecular mechanism underlying microtubule-lattice heterogeneity.

Illustration of the different modes of tubulin association with microtubule growing ends. Red dots indicate new interactions. (A) Formation of multiple seams. 1) Unique longitudinal interaction. 2) Combined lateral and longitudinal interactions. 3) Unique lateral interaction between one $\alpha$-tubulin subunit of an incoming tubulin dimer and a $\beta$-tubulin subunit at the tip of the growing microtubule. 4-5) Incorporation of the lattice-type transitions inside the microtubule wall during microtubule elongation. Two A-lattice seams have been formed (fine arrows). (B) Incorporation of a tubulin dimer gap without change in lattice type organization. 1) Homotypic lateral interaction of an incoming tubulin dimer without longitudinal interaction. 2-5) Incorporation of a tubulin dimer gap inside the microtubule lattice. (C) Lateral offset of the seam by one protofilament during elongation. 1) Unique longitudinal interaction. 2) Homotypic interaction of an incoming dimer at the seam region without longitudinal contacts. 3-4) Incorporation of a lattice-type transition inside the microtubule wall. The seam has moved laterally by one protofilament (4), a situation systematically encountered in cytoplasmic Xenopus egg-extract microtubules.

\section{Acknowledgments:}

Cryo-electron microscopy data were acquired on the Microscopy Rennes imaging center platform (Biosit, Rennes, France), member of the national infrastructure FranceBioImaging (FBI) supported by the French National Research Agency (ANR-10-INBS-04).

Xenopus laevis eggs were obtained from the Centre de Ressources Biologique Xénopes, University or Rennes 1, Rennes, France.

Porcine brains were kindly provided by Y. Drillet, Cooperl Arc Altantique, Lamballe France.

Tobacco Mosaic Virus was kindly provided by T. Candresse, UMR 13332 Biologie du Fruit et Pathologie, INRAE and University of Bordeaux, Villenave d'Ornon, France.

\section{Funding:}

French National Research Agency ANR-16-CE11-0017-01 (MOS, DC)

French National Research Agency ANR-18-CE13-0001-01 (DC)

Swiss National Science Foundation 310030_192566 (MOS)

Human Frontier Science Program CDA00019/2019-C (RG)

\section{Author contributions:}

Conceptualization: MOS, RG, DC

Funding acquisition: MOS, RG, DC

Investigation: $\mathrm{CG}, \mathrm{SK}, \mathrm{GG}, \mathrm{RG}, \mathrm{DC}$

Methodology: CG, RG, DC

Project administration: DC

Resources: NO, CH, LD

Software: JH, CB 
Supervision: MOS, RG, DC

Visualization: CG, DC

Writing - original draft: CG, DC

Writing - review \& editing: MOS, RG, DC

Competing interests: Authors declare that they have no competing interests.

Data and materials availability: All numerical data are available in the main text or the supplementary materials. Dual-axis cryo-electron tomograms and sub-tomogram averages with their associated models are available from the corresponding author on request. 